\title{
Patient Error: A Preliminary Taxonomy
}

Stepben Buetow, $\mathrm{PbD}^{1}$

Liz Kiata, $M A^{2}$

Tess Liew, $M A^{2}$

Tim Kenealy, $P b D, M B C b B^{1}$

Susan Dovey, $P b D^{3}$

Glyn Elwyn, $\mathrm{MBBCb}, \mathrm{PbD}^{4}$

'Department of General Practice and Primary Health Care, University of Auckland, Auckland, New Zealand

${ }^{2}$ Department of Social and Community Health, University of Auckland, Auckland, New Zealand

${ }^{3}$ Department of General Practice, University of Otago, Dunedin, New Zealand

${ }^{4}$ Department of Primary Care and Public Health, Cardiff University, Wales

Conflicts of interest: none reported

\section{CORRESPONDING AUTHOR}

Stephen Buetow, PhD

Department of General Practice and Primary Health Care

University of Auckland

Private Bag 92019

Auckland Mail Centre

Auckland 1142, New Zealand

s.buetow@auckland.ac.nz

\begin{abstract}
PURPOSE Current research on errors in health care focuses almost exclusively on system and clinician error. It tends to exclude how patients may create errors that influence their health. We aimed to identify the types of errors that patients can contribute and help manage, especially in primary care.
\end{abstract}

METHODS Eleven nominal group interviews of patients and primary health care professionals were held in Auckland, New Zealand, during late 2007. Group members reported and helped to classify types of potential error by patients. We synthesized the ideas that emerged from the nominal groups into a taxonomy of patient error.

RESULTS Our taxonomy is a 3-level system encompassing 70 potential types of patient error. The first level classifies 8 categories of error into 2 main groups: action errors and mental errors. The action errors, which result in part or whole from patient behavior, are attendance errors, assertion errors, and adherence errors. The mental errors, which are errors in patient thought processes, comprise memory errors, mindfulness errors, misjudgments, and-more distallyknowledge deficits and attitudes not conducive to health.

CONCLUSION The taxonomy is an early attempt to understand and recognize how patients may err and what clinicians should aim to influence so they can help patients act safely. This approach begins to balance perspectives on error but requires further research. There is a need to move beyond seeing patient, clinician, and system errors as separate categories of error. An important next step may be research that attempts to understand how patients, clinicians, and systems interact to cocreate and reduce errors.

Ann Fam Med 2009;7:223-231. DOI: 10.1370/afm.941.

\section{INTRODUCTION}

M ost literature on medical error focuses on clinician and system error in clinical settings including hospitals ${ }^{1,2}$ and primary care..$^{3,4}$ The medical errors in primary care have been defined as events "in your practice that should not have happened." ${ }^{15-7}$ This focus emphasizes the health care facilities where clinicians can err. They may err at the front line (active errors) or through system deficiencies outside their direct control (latent errors). Taxonomies of medical error reflect this locationcentric approach. ${ }^{4-10}$

Where does the patient fit? Although patients have reported clinical problems in primary care ${ }^{11}$ and hospitals, ${ }^{12}$ the lack of attention to the patient perspective has been criticized. ${ }^{13}$ Moreover, patients and their caregivers "make errors too."14(p33) With some exceptions, ${ }^{15-19}$ however, patients' contribution to their own suboptimal health ${ }^{20,21}$ has not usually been conceptualized as error. Almost all the taxonomies of medical error, ${ }^{4-6,8,9,22}$ do not discuss patients' contribution to error, or they acknowledge this contribution peripherally, ${ }^{23}$ perhaps because patient error is a sensitive issue. It is easy to confuse human error with blame and to view patients, in particular, as incapable of error because they can be sick and tend to have reduced power in their interactions with clinicians and the health system. 
Yet we respect-not disrespect-competent patients by acknowledging their capacity, as people, to make choices and err. Furthermore, although patients' contribution to error is unknown, by far the largest group providing health care is patients themselves ${ }^{24}$; and it is likely "that the number of opportunities to reduce medical errors in different parts of the health system is proportional to the number of patient contacts made there." 25 Self-care and primary care characterize most patient contacts ${ }^{24}$ and probably, therefore, most patient errors

We have previously explored the concept and context of patient error, including the factors that can predispose patients to err. ${ }^{26}$ For us, patient errors are primarily patient actions that (1) are not completed as the patient intended (errors of execution) or (2) do not achieve the outcome that the patient intended because the plan was not based on informed and strong patient beliefs (errors of planning). This definition builds on work by Reason. ${ }^{27}$ Second, these errors may be preceded by potential errors along cognitive dimensions. ${ }^{28}$

From our perspective, intended nonadherence is not an error if the plan is rational to the patient ${ }^{29}$ and achieves the outcome that the patient intended. For example, a patient might choose not to access a recommended screening program because it would take time from work and, aware of the cost-benefits of attendance, might deem the cost to be too high. ${ }^{30}$ Nonadherence in this case is not an error if the intended outcome is not to be screened, and the patient understands the risks and makes the decision freely.

Supported by this conceptualization, our study aimed to address the paucity of empirical work on patient error by beginning the tasks of exploring and classifying the types of error that patients can contribute. Complementing classifications of medical error ${ }^{22,28,31}$ in nursing ${ }^{32}$ and family practice, ${ }^{4-10}$ we wanted to construct a taxonomy of patient error that could organize patient and professional perspectives on how patients can err and when. We wanted to elicit these perspectives in structured group activities involving patients in a community setting and primary care professionals.

\section{METHODS}

\section{Nominal Group Technique}

The nominal group technique was used to collect our data. This technique is a highly structured application of small-group discussion methods. It engages a nominal group - a group in name only - in generating anonymous ideas that participants share with each other. We used the nominal group technique to help all group members stay focused on and complete the tasks of exploring the types of errors that patients can make. ${ }^{33}$ The perspectives of all participants carry equal weight, the approach is transparent, and it facilitates both immediate feedback to the research team and social interaction as stimuli to idea generation. ${ }^{34}$

\section{Sampling}

We purposively selected 11 homogenous nominal groups to enhance variation in the ability of our sample to represent patient error from different perspectives. To emphasize patient perspectives, we wanted 8 of the groups to be patient groups. The other 3 groups comprised the types of primary care professionals whom patients commonly visit.

Two community-based organizations recruited the groups of patients with known or presumed characteristics, mostly from a suburban community of low socioeconomic status. We drew on our professional networks to recruit the professional groups.

To be eligible for selection, each group participant needed to be able to speak conversational English; have used, or provided, formal health care during the previous 5 years; be a member of the group sampled (eg, the women's group); be independent of other participants in the same group $;$ have reported that he or she could contribute usefully to our study on the basis of knowledge and experience; and be willing and able to give written, informed consent to participate. Interested persons were recruited, or not, after we assessed that person's suitability for participation. Group meetings with the patients were held in community settings. The meetings with health professionals were held at the university. This study received ethics approval from the Northern Region Ethics Committee of New Zealand.

\section{Data Gathering}

All the meetings were conducted in English, audio recorded and co-facilitated by 2 authors (S.B., L.K.) in late 2007. One facilitator managed the group process; the other observed how the group interacted and gave administrative and technical support. After all the participants had read the information sheet and given written consent to take part in the project, the facilitators introduced themselves. They explained the purpose of the project, including the opportunity to improve patient safety by identifying without blame the types of errors that patients can contribute. They described how the meetings would be conducted to achieve the study aim while protecting participants' rights, such as to be treated with respect by everyone present. Participants then introduced themselves.

The question posed to each group was, "What mistakes can patients make?" Mistakes were not distinguished from errors for our participants. Also, the concepts of "patient" and "mistake" were not explicitly 
defined for or by the participants. The facilitator, however, offered examples of patient mistakes, such as forgetting to attend for an appointment. How participants then conceptualized mistakes was revealed by the types of mistakes they identified. They were told they could draw on their own mistakes or mistakes they knew others had made. They could also imagine the mistakes that patients can make.

To answer our question, participants first engaged in the silent, independent, and anonymous generation of ideas in writing. On individual $9 \times 11$-inch sheets of paper, they each wrote these ideas, 1 per sheet, in as few words as possible in large text. Round-robin recording of these ideas followed. Participants were each encouraged to give a facilitator 1 completed sheet, which was affixed to the wall during each round and read aloud. Participants used ideas from other participants to write down further thoughts. If someone else offered the same idea a participant had written down but not yet contributed, that participant did not need to share it. If an idea were even slightly different, however, the participant was encouraged to offer it to the group. Participants could pass on any given round and return on a later round. Group discussion followed when it was necessary to make clear the meaning of the idea and the reason for including it. Any member could clarify or explain ideas so as to protect the anonymity of the individuals who contributed them. These processes continued until all the ideas had been collected. When an idea was repeated, it was possible to remove the duplicate. Next, categorization took place as participants worked together to put ideas into common groupings. We explained that we intended to combine the results from all the nominal groups, report back to the community, and make a written summary of the results available to them at that time. The meetings lasted approximately 2 hours.

\section{Analysis}

We used a general inductive approach $^{35}$ to combine and categorize the lists of potential errors reported by each nominal group and group discussion to help clarify and express items during the meetings. The primary author (S.B.) first imported the lists into QSR NVivo, a software program for managing and supporting the analysis of qualitative data (NVivo, version 1.2, QSR International Pty Ltd, Doncaster, Victoria, Australia). He closely read the lists sev- eral times to become familiar with their content and core meanings; coded the individual errors that patients could make; and categorized the errors on the basis of emergent themes that were consistent with the study aim. The categorization was made transparent by constructing levels to clarify how the final categories emerged through abstraction of the specific errors suggested by study participants; the same usage of levels also characterizes some other taxonomies of medical error in primary care ${ }^{5-7}$ and may assist the replication, validation, and further development of our work. Relations between the categories were identified to reveal the temporal sequence of the category system. The other team members scrutinized and amended the taxonomy, as did participants from our nominal groups when we fed back results to them at 2 meetings during early 2008. We compared the types of errors suggested by patients and health professionals, respectively, but our primary focus was on pooling the errors suggested by all the groups.

\section{RESULTS}

Table 1 describes the age and sex composition of our sample of 8 patient groups and 3 professional groups. The number of participants in these 11 groups ranged from 5 to 9 , and averaged 7 . The total number of participants was 83, including 64 patients. The patient group was disproportionately female (72\%) and approximately three-fifths of the participants in both the patient and professional groups were aged 20 to 54 years.

From the perspective of this sample, analysis of the self-report data yielded a taxonomy of errors that patients can make (Table 2). The taxonomy is a 3-level

\begin{tabular}{|c|c|c|c|c|c|}
\hline Group & No. & $\begin{array}{c}\text { Female } \\
\text { No. }\end{array}$ & $\begin{array}{c}\text { Age }<20 \text { y } \\
\text { No. }\end{array}$ & $\begin{array}{c}\text { Age } 20-54 \text { y } \\
\text { No. }\end{array}$ & $\begin{array}{c}\text { Age } \geq 55 \text { y } \\
\text { No. }\end{array}$ \\
\hline \multicolumn{6}{|l|}{ Patient groups } \\
\hline $\begin{array}{l}\text { 1. English as a second } \\
\text { language }\end{array}$ & 9 & 7 & 0 & 5 & 4 \\
\hline 2. High health literacy & 5 & 5 & 0 & 5 & 0 \\
\hline 3. Independent elders & 8 & 4 & 0 & 2 & 6 \\
\hline 4. Informal carergivers & 8 & 8 & 0 & 7 & 1 \\
\hline 5. Men & 9 & 0 & 0 & 5 & 4 \\
\hline 6. Māori & 8 & 8 & 1 & 5 & 2 \\
\hline 7. Teenagers & 8 & 5 & 8 & 0 & 0 \\
\hline 8. Women & 9 & 9 & 0 & 7 & 2 \\
\hline Total & 64 & 46 & 9 & 36 & 19 \\
\hline \multicolumn{6}{|l|}{ Professional groups } \\
\hline 9. Family physicians & 5 & 1 & 0 & 4 & 1 \\
\hline 10. Practice nurses & 7 & 7 & 0 & 4 & 3 \\
\hline 11. Pharmacists & 7 & 2 & 0 & 4 & 3 \\
\hline Total & 19 & 10 & 0 & 12 & 7 \\
\hline
\end{tabular}


Table 2. Taxonomy of Patient Error

\begin{tabular}{|c|c|c|c|c|}
\hline \multirow[b]{2}{*}{ Level 1} & \multirow[b]{2}{*}{ Level 2} & \multirow[b]{2}{*}{ Level 3} & \multicolumn{2}{|l|}{ Examples $^{a}$} \\
\hline & & & Type of Error & Participant Comment \\
\hline \multicolumn{5}{|l|}{ Action errors } \\
\hline \multirow[t]{11}{*}{$\begin{array}{l}\text { 1. Attendance } \\
\text { errors }\end{array}$} & $\begin{array}{l}\text { 1.1 Underatten- } \\
\text { dance }\end{array}$ & 1.1.1 Nonattendance & Refusal of visits & $\begin{array}{l}\text { "If it runs through the family we seek } \\
\text { no help" (group 2) }\end{array}$ \\
\hline & & $\begin{array}{l}\text { 1.1.2 Insufficient } \\
\text { attendance }\end{array}$ & $\begin{array}{l}\text { Less frequent attendance than } \\
\text { recommended }\end{array}$ & $\begin{array}{l}\text { "(Not) following your gut instinct and not } \\
\text { seeking a second opinion" (group } 8 \text { ) }\end{array}$ \\
\hline & $\begin{array}{l}\text { 1.2 Untimely } \\
\text { attendance }\end{array}$ & 1.2.1 Early attendance & $\begin{array}{l}\text { Attendance for self-limiting } \\
\text { conditions }\end{array}$ & "Hypochondriacal behavior" (group 5) \\
\hline & & 1.2.2 Late attendance & Deferral of visit & $\begin{array}{l}\text { "Delay in visiting the doctor when you } \\
\text { know you're sick" (group 2) }\end{array}$ \\
\hline & $\begin{array}{l}\text { 1.3 Overatten- } \\
\text { dance }\end{array}$ & $\begin{array}{l}\text { 1.3.1 Frequent } \\
\text { attendance }\end{array}$ & $\begin{array}{l}\text { More frequent attendance } \\
\text { than required }\end{array}$ & $\begin{array}{l}\text { "Overattendance (eg, because the doc- } \\
\text { tor is cute)" (group 2) }\end{array}$ \\
\hline & 1.4 Misattendance & $\begin{array}{l}\text { 1.4.1 Inappropriate } \\
\text { type of visit }\end{array}$ & $\begin{array}{l}\text { Demand for a home visit by } \\
\text { a patient who could have } \\
\text { safely come to the clinic }\end{array}$ & Irregularity (group 2) \\
\hline & & $\begin{array}{l}\text { 1.4.2 No usual pro- } \\
\text { vider chosen }\end{array}$ & Frequently changing providers & "Consulting multiple doctors" (group 9) \\
\hline & & $\begin{array}{l}\text { 1.4.3 Use of unquali- } \\
\text { fied sources }\end{array}$ & $\begin{array}{l}\text { Use of unqualified comple- } \\
\text { mentary sources }\end{array}$ & $\begin{array}{l}\text { Taking advice from marginal sources: } \\
\text { "over the fence, nonqualified practi- } \\
\text { tioners, TV adverts" (group 9) }\end{array}$ \\
\hline & & $\begin{array}{l}\text { 1.4.4. Refusals during } \\
\text { visits }\end{array}$ & $\begin{array}{l}\text { Refusal to be examined by a } \\
\text { student doctor }\end{array}$ & $\begin{array}{l}\text { "Refusing to be checked by the doctor" } \\
\text { (group 1) }\end{array}$ \\
\hline & & $\begin{array}{l}\text { 1.4.5 No escort when } \\
\text { needed }\end{array}$ & No interpreter & $\begin{array}{l}\text { "Not coming in with an interpreter } \\
\text { (friend/relative) when their communica- } \\
\text { tion in English is suboptimal" (group 9) }\end{array}$ \\
\hline & & $\begin{array}{l}\text { 1.4.6 Inappropriate } \\
\text { escort/chaperone }\end{array}$ & Child & $\begin{array}{l}\text { "Not telling doctor what their real con- } \\
\text { cerns are" (group 9) }\end{array}$ \\
\hline \multirow[t]{12}{*}{$\begin{array}{l}\text { 2. Assertion } \\
\text { errors }\end{array}$} & 2.1 Taciturnity & $\begin{array}{l}\text { 2.1.1 Nondisclosure of } \\
\text { relevant information }\end{array}$ & $\begin{array}{l}\text { Not updating contact } \\
\text { information }\end{array}$ & $\begin{array}{l}\text { "Not telling the doctor all your symp- } \\
\text { toms" (group 7) }\end{array}$ \\
\hline & & 2.1.2 Nonquestioning & $\begin{array}{l}\text { Not asking for clarification of } \\
\text { confusing information }\end{array}$ & $\begin{array}{l}\text { "Not questioning professionals if } \\
\text { instructions are unclear or they do not } \\
\text { understand" (group 10) }\end{array}$ \\
\hline & 2.2 Verbosity & 2.2.1 Excessive talk & $\begin{array}{l}\text { Not giving the clinician suffi- } \\
\text { cient time to meet concerns }\end{array}$ & $\begin{array}{l}\text { "Telling doctor what I want but not giv- } \\
\text { ing much time for him to tell me what } \\
\text { he would like" (group 3) }\end{array}$ \\
\hline & $\begin{array}{l}\text { 2.3 Extraneous } \\
\text { talk }\end{array}$ & 2.3.1 Irrelevant talk & $\begin{array}{l}\text { Trying too hard to recall } \\
\text { details }\end{array}$ & $\begin{array}{l}\text { "No relationship with doctor, so just say } \\
\text { 'yes' to everything" (group 1) }\end{array}$ \\
\hline & 2.4 Erroneous talk & 2.4.1 Inaccurate talk & $\begin{array}{l}\text { Contradicting medical advice } \\
\text { to family or friends }\end{array}$ & "Inaccurate/false responses" (group 2) \\
\hline & $\begin{array}{l}2.5 \\
\text { Inarticulateness }\end{array}$ & $\begin{array}{l}\text { 2.5.1 Inability to express } \\
\text { thoughts clearly }\end{array}$ & $\begin{array}{l}\text { Limited language skills; trans- } \\
\text { lation errors. }\end{array}$ & $\begin{array}{l}\text { Inability to describe your sickness" } \\
\text { (group 7) }\end{array}$ \\
\hline & 2.6 Disrespect & 2.6.1 Lack of caring & $\begin{array}{l}\text { Lack of regard for interests of } \\
\text { clinician }\end{array}$ & $\begin{array}{l}\text { "Making } 1 \text { appointment for } 2 \text { to } 4 \\
\text { people" (group 10) }\end{array}$ \\
\hline & & 2.6.2 Discourtesy & Cell phone on during visits & $\begin{array}{l}\text { "Not notifying if late or, need to miss, } \\
\text { appointments" (group 10) }\end{array}$ \\
\hline & & 2.6.3 Abusiveness & Violent patient & "Being drunk and abusive" (group 6) \\
\hline & 2.7 Artfulness & 2.7.1 Dishonesty & $\begin{array}{l}\text { Distortion of information } \\
\text { given }\end{array}$ & $\begin{array}{l}\text { "Lying about symptoms to jump queue" } \\
\text { (group 4) }\end{array}$ \\
\hline & & $\begin{array}{l}\text { 2.7.2 Pretense of } \\
\text { sickness }\end{array}$ & Benefits of sick role & $\begin{array}{l}\text { "Pretending to be ill to take the day off } \\
\text { school" (group 7) }\end{array}$ \\
\hline & & $\begin{array}{l}\text { 2.7.3 Manipulation of } \\
\text { system }\end{array}$ & False claims for compensation & $\begin{array}{l}\text { "Seeks to manipulate the outflow of } \\
\text { information from the medical record" } \\
\text { (group 9) }\end{array}$ \\
\hline \multirow[t]{6}{*}{$\begin{array}{l}\text { 3. Adherence } \\
\text { errors }\end{array}$} & $\begin{array}{l}3.1 \text { Collection } \\
\text { errors }\end{array}$ & $\begin{array}{l}\text { 3.1.1 Prescriptions not } \\
\text { redeemed }\end{array}$ & $\begin{array}{l}\text { Prescribed medications not } \\
\text { collected from pharmacies }\end{array}$ & $\begin{array}{l}\text { "Only getting medications they can } \\
\text { afford for now" (group 10) }\end{array}$ \\
\hline & 3.2 Storage errors & 3.2.1 Storage errors & $\begin{array}{l}\text { Storage of medications past } \\
\text { expiration date }\end{array}$ & $\begin{array}{l}\text { "Accumulating discontinued medica- } \\
\text { tions" (group 9) }\end{array}$ \\
\hline & $\begin{array}{l}3.3 \text { Self-adminis- } \\
\text { tration }\end{array}$ & 3.3.1 No treatment & $\begin{array}{l}\text { Failure to take recommended } \\
\text { treatment }\end{array}$ & "Running out of medications" (group 10) \\
\hline & & 3.3.2 Wrong treatment & Taking discontinued treatment & "Using old medication" (group 6) \\
\hline & & 3.3.3 Dosage errors & Excessive dosage & $\begin{array}{l}\text { "Doubling up treatment if going away" } \\
\text { (group 3) }\end{array}$ \\
\hline & & & & Table 2 continues \\
\hline
\end{tabular}


Table 2. Taxonomy of Patient Error (continued)

\begin{tabular}{|c|c|c|c|c|}
\hline \multirow[b]{2}{*}{ Level 1} & \multirow[b]{2}{*}{ Level 2} & \multirow[b]{2}{*}{ Level 3} & \multicolumn{2}{|l|}{ Examples $^{a}$} \\
\hline & & & Type of Error & Participant Comment \\
\hline \multicolumn{5}{|c|}{ Action errors (continued) } \\
\hline & & 3.3.4 Timing errors & $\begin{array}{l}\text { Taking medication at incorrect } \\
\text { times }\end{array}$ & $\begin{array}{l}\text { "Taking medication in wrong order" } \\
\text { (group 11) }\end{array}$ \\
\hline & & 3.3.5 Duration errors & $\begin{array}{l}\text { Treatment duration is shorter } \\
\text { than recommended }\end{array}$ & "Stopping too soon" (group 11) \\
\hline & & $\begin{array}{l}\text { 3.3.6 Hazardous } \\
\text { interactions }\end{array}$ & $\begin{array}{l}\text { Interactions of over-the-counter } \\
\text { and prescribed treatments }\end{array}$ & "Mixing pills and alcohol" (group 6) \\
\hline & $\begin{array}{l}\text { 3.4 Other delivery } \\
\text { errors }\end{array}$ & $\begin{array}{l}\text { 3.4.1 Sharing of } \\
\text { medication }\end{array}$ & $\begin{array}{l}\text { Sharing medication with fam- } \\
\text { ily or friends }\end{array}$ & $\begin{array}{l}\text { "Sharing resources with relatives, eg, } \\
\text { 'rescue' asthma inhalers" (group 10) }\end{array}$ \\
\hline \multicolumn{5}{|c|}{ Mental errors: proximate determinants } \\
\hline \multirow[t]{2}{*}{$\begin{array}{l}\text { 4. Memory } \\
\text { errors }\end{array}$} & 4.1 Memory lapses & 4.1.1 Forgetfulness & Forgetting to take medication & $\begin{array}{l}\text { "Forgetting to collect the medication" } \\
\text { (group 11) }\end{array}$ \\
\hline & & $\begin{array}{l}\text { 4.1.2 Misrecalling } \\
\text { information }\end{array}$ & $\begin{array}{l}\text { Misrecalling when to attend } \\
\text { for care }\end{array}$ & "Turn up at wrong time" (group 1) \\
\hline \multirow[t]{3}{*}{$\begin{array}{l}\text { 5. Mindfulness } \\
\text { errors }\end{array}$} & 5.1 Inattention & 5.1.1 Failure to notice & Not perceiving & $\begin{array}{l}\text { "Not listening to what the doctor says" } \\
\text { (group 8) }\end{array}$ \\
\hline & & $\begin{array}{l}\text { 5.1.2 Recognize } \\
\text { incorrectly }\end{array}$ & Misreading of symptoms & $\begin{array}{l}\text { "Over-reacting to children's symptoms" } \\
\text { (group 4) }\end{array}$ \\
\hline & $\begin{array}{l}\text { 5.2 Overattentive- } \\
\text { ness }\end{array}$ & 5.2.1 Hypervigilance & $\begin{array}{l}\text { Overattentiveness to variations } \\
\text { in normal function }\end{array}$ & "Hyperchondriacal behavior" (group 5) \\
\hline \multirow[t]{8}{*}{ 6. Misjudgments } & $\begin{array}{l}6.1 \text { Assessment } \\
\text { errors }\end{array}$ & 6.1.1 Failure to check & $\begin{array}{l}\text { Failure to check on laboratory } \\
\text { results }\end{array}$ & $\begin{array}{l}\text { "Not checking pills from chemist" } \\
\text { (group 3) }\end{array}$ \\
\hline & & $\begin{array}{l}\text { 6.1.2 Failure to } \\
\text { monitor }\end{array}$ & Failure to monitor weight & $\begin{array}{l}\text { "Not monitoring blood glucose as rec- } \\
\text { ommended" (group 10) }\end{array}$ \\
\hline & & 6.1.3 Failure to record & $\begin{array}{l}\text { Failure to keep a patient diary } \\
\text { when requested }\end{array}$ & $\begin{array}{l}\text { "Not recording symptoms when asked, } \\
\text { or bringing record back as asked" } \\
\text { (group 9) }\end{array}$ \\
\hline & & $\begin{array}{l}\text { 6.1.4 Wrong } \\
\text { assessment }\end{array}$ & Misreading of instructions & $\begin{array}{l}\text { "Stopping medication just because you } \\
\text { feel better" (group 1) }\end{array}$ \\
\hline & $\begin{array}{l}6.2 \text { Unrealistic } \\
\text { expectations }\end{array}$ & $\begin{array}{l}\text { 6.2.1 Overexpectation } \\
\text { of others }\end{array}$ & Immediate cure & $\begin{array}{l}\text { "Expecting the doctor to read their } \\
\text { mind" (group 9) }\end{array}$ \\
\hline & & $\begin{array}{l}\text { 6.2.2 Overexpectation } \\
\text { of self }\end{array}$ & Self-diagnosis & $\begin{array}{l}\text { "Using the Internet for self-diagnosis } \\
\text { and self-treatment" (group 10) }\end{array}$ \\
\hline & & $\begin{array}{l}\text { 6.2.3 Underexpecta- } \\
\text { tion of others }\end{array}$ & $\begin{array}{l}\text { Expected inability of clinician } \\
\text { to help }\end{array}$ & "Having no faith in doctors" (group 8) \\
\hline & & $\begin{array}{l}\text { 6.2.4 Underexpecta- } \\
\text { tion of self }\end{array}$ & $\begin{array}{l}\text { Expected inability of self to } \\
\text { cope or share responsibilities }\end{array}$ & $\begin{array}{l}\text { "Inability to cope with new presenta- } \\
\text { tions" (group 11) }\end{array}$ \\
\hline \multicolumn{5}{|c|}{ Memory errors: background determinants } \\
\hline \multirow[t]{5}{*}{$\begin{array}{l}\text { 7. Knowledge } \\
\text { deficits }\end{array}$} & $\begin{array}{l}7.1 \text { Knowledge } \\
\text { errors }\end{array}$ & 7.1.1 Low literacy & Poor language skills & $\begin{array}{l}\text { "Inability to read and understand } \\
\text { instructions" (group 1) }\end{array}$ \\
\hline & & $\begin{array}{l}\text { 7.1.2 Low health } \\
\text { literacy }\end{array}$ & $\begin{array}{l}\text { Not knowing the name of } \\
\text { medications }\end{array}$ & $\begin{array}{l}\text { "Confusion over brand, shape, color and } \\
\text { name (especially when these change" } \\
\text { (group 11) }\end{array}$ \\
\hline & & 7.1.3 Low numeracy & Inability to budget & $\begin{array}{l}\text { "Not budgeting and not having an } \\
\text { emergency fund for medical care" } \\
\text { (group 8) }\end{array}$ \\
\hline & $\begin{array}{l}\text { 7.2 Comprehen- } \\
\text { sion errors }\end{array}$ & $\begin{array}{l}\text { 7.2.1 Lack of } \\
\text { understanding }\end{array}$ & $\begin{array}{l}\text { Failure to understand } \\
\text { instructions }\end{array}$ & $\begin{array}{l}\text { "Not understanding instructions (eg, re: } \\
\text { casts, equipment)" (group 10) }\end{array}$ \\
\hline & 7.3 Logic errors & 7.3.1 Reasoning errors & $\begin{array}{l}\text { Considering that a medication } \\
\text { imparts absolute protection }\end{array}$ & $\begin{array}{l}\text { "Assuming that must be OK because } \\
\text { feeling good" (group 2) }\end{array}$ \\
\hline \multirow{5}{*}{$\begin{array}{l}\text { 8. Attitudes not } \\
\text { conducive to } \\
\text { health }\end{array}$} & 8.1 Selfishness & 8.3.1 Excessive pride & $\begin{array}{l}\text { Reluctance to ask for, or } \\
\text { accept help }\end{array}$ & $\begin{array}{l}\text { "Reluctance to ask for help (eg, credit) } \\
\text { because of pride" (group 8) }\end{array}$ \\
\hline & & 8.3.2 Dishonesty & Lying & $\begin{array}{l}\text { "Lying about symptoms to jump queue" } \\
\text { (group 4) }\end{array}$ \\
\hline & & 8.3.3 Self-pity & Feeling a victim & $\begin{array}{l}\text { "Feeling self-pity; becoming a victim" } \\
\text { (group 1) }\end{array}$ \\
\hline & & 8.3.4 Hedonism & $\begin{array}{l}\text { Willingness to drink alcohol } \\
\text { inappropriately }\end{array}$ & $\begin{array}{l}\text { "Taking medicines for recreational use } \\
\text { (eg, too much insulin to get a high)" } \\
\text { (group 11) }\end{array}$ \\
\hline & & & & Table 2 continues \\
\hline
\end{tabular}




\begin{tabular}{|c|c|c|c|c|}
\hline \multirow[b]{2}{*}{ Level 1} & \multirow[b]{2}{*}{ Level 2} & \multirow[b]{2}{*}{ Level 3} & \multicolumn{2}{|l|}{ Examples $^{\mathrm{a}}$} \\
\hline & & & Type of Error & Participant Comment \\
\hline \multicolumn{5}{|c|}{ Memory errors: background determinants (continued) } \\
\hline & \multirow[t]{4}{*}{8.2 Self-neglect } & $\begin{array}{l}\text { 8.2.1 Excessive } \\
\text { selflessness }\end{array}$ & $\begin{array}{l}\text { Carriage of other people's } \\
\text { burdens }\end{array}$ & $\begin{array}{l}\text { "Putting other people's needs first" } \\
\text { (group 2) }\end{array}$ \\
\hline & & $\begin{array}{l}\text { 8.2.2 Lack of } \\
\text { self-regard }\end{array}$ & Shyness at visits & $\begin{array}{l}\text { "Forget to love oneself; putting other } \\
\text { people before yourself" (group 4) }\end{array}$ \\
\hline & & 8.2.3 Carelessness & Carelessness & "Losing instructions" (group 2) \\
\hline & & 8.2.4 Embarrassment & Shame & $\begin{array}{l}\text { "Not taking medications because you } \\
\text { think your friends might mock you" } \\
\text { (group 7) }\end{array}$ \\
\hline & \multirow[t]{5}{*}{8.3 Carelessness } & 8.4.1 Inattention & $\begin{array}{l}\text { Distractedness or } \\
\text { absent-mindedness }\end{array}$ & $\begin{array}{l}\text { "Patient distracted-not engaging in } \\
\text { the consultation" (group 10) }\end{array}$ \\
\hline & & $\begin{array}{l}\text { 8.4. } 2 \text { Thoughtlessness } \\
\text { regarding others }\end{array}$ & $\begin{array}{l}\text { Sharing food and drink while } \\
\text { infectious }\end{array}$ & $\begin{array}{l}\text { "Not staying home when feeling sick (so } \\
\text { spreading influenza)" (group 8) }\end{array}$ \\
\hline & & $\begin{array}{l}\text { 8.4.3 Excessive risk } \\
\text { taking }\end{array}$ & "She'll be OK" attitude & $\begin{array}{l}\text { "Taking risks with your health when } \\
\text { sick" (group 5) }\end{array}$ \\
\hline & & 8.4.4 Apathy & Laziness in getting medication & $\begin{array}{l}\text { "Noncollection of medicines because of } \\
\text { sloth" (group 11) }\end{array}$ \\
\hline & & 8.4.5 Unreliability & $\begin{array}{l}\text { Inconsistency in passing on } \\
\text { messages }\end{array}$ & "Inconsistent with medication" (group 3) \\
\hline & \multirow[t]{4}{*}{ 8.5 Distrust } & 8.5.1 Disbelief & $\begin{array}{l}\text { Suspicion of health } \\
\text { professionals }\end{array}$ & "Not believing the doctor" (group 2) \\
\hline & & 8.5.2 Fearfulness & Fear of needles & $\begin{array}{l}\text { "Staying with an unhelpful doctor } \\
\text { because of familiarity and fear of } \\
\text { change" (group 1) }\end{array}$ \\
\hline & & $\begin{array}{l}\text { 8.5.3 Uncooperative- } \\
\text { ness }\end{array}$ & Unwillingness to negotiate & $\begin{array}{l}\text { "Refusing to be checked by the doctor" } \\
\text { (group 1) }\end{array}$ \\
\hline & & 8.5.4 Pessimism & Feeling of helplessness & "Giving up hope" (group 1) \\
\hline & \multirow[t]{2}{*}{ 8.6 Anger } & 8.6.1 Impatience & $\begin{array}{l}\text { Impatience while waiting for } \\
\text { care }\end{array}$ & $\begin{array}{l}\text { "Not having patience while waiting" } \\
\text { (group 6) }\end{array}$ \\
\hline & & 8.6.2 Intolerance & $\begin{array}{l}\text { Prejudice against doctors } \\
\text { with non-English speaking } \\
\text { backgrounds }\end{array}$ & $\begin{array}{l}\text { "Stressing out on things that you have } \\
\text { forgotten to do" (group 4) }\end{array}$ \\
\hline & $\begin{array}{l}\text { 8.7 Other } \\
\text { priorities }\end{array}$ & 8.7.1 Cultural priorities & $\begin{array}{l}\text { Mourning takes priority over } \\
\text { medication adherence }\end{array}$ & $\begin{array}{l}\text { "Tangi [funeral] disrupts medical/health } \\
\text { needs" (group 3) }\end{array}$ \\
\hline
\end{tabular}

system encompassing 70 errors in 8 broad categories that reduce to 2 domains: (1) action errors and (2) mental errors. Patient action errors are errors resulting, in part or whole, from patient behavior. Patient mental errors are errors in patient thought processes.

The mental errors logically precede the action errors in the chain of patient safety events. We distinguish between the mental errors that are most proximate to the action error and background mental errors The table makes clear the level-2 and level-3 errors from which we derived the 8 level-1 categories. Examples are given of each of the level-3 errors with which we began our analysis, using both our own words and participants' words. This audit trail supports the transparency and trustworthiness of the analysis.

\section{Action Errors}

The taxonomy reports 3 sets of action errors that patients can make: attendance errors, assertion errors, and adherence errors. Attendance errors are errors in the number, timing, and type of visits. These errors include underattendance in the forms of nonattendance and insufficient attendance. Patients may also err through attendance that is excessive or untimely (early or late) in the appearance of the problem or the time of arrival for visits. Misattendance can result from inappropriate (eg, irregular) visits; not visiting a usual or qualified provider; refusals to receive care, eg, from a student doctor; and an inappropriate escort, such as a child, or no escort when required.

Assertion errors are patient errors in communicating with and relating to providers. These errors include the amount, content, and method of patient communication. Patients may err by saying too little or too much during interactions. What they talk about may be inaccurate or unhelpful to the provider. Patients might not convey the message they intend if they communicate unclearly, with disrespect, or artfully. Forms of artfulness include dishonesty, pretence of sickness, and manipulating the system, as when patients aim "to manipulate the outflow of information from the medical record." Patients and health care 
professionals alike reported patient errors of attendance and assertion.

Adherence errors are patient errors over time in abiding by the regimen of treatment, especially medication. They pertain to errors in patients' collection, storage, and self-administration of treatments. The last errors take diverse forms, including unintended nonadherence, adherence to the wrong treatment; errors relating to the timing, dosage, duration, and interactions of treatments, such as "mixing pills and alcohol"; and other delivery errors, such as "sharing resources with relatives, eg, 'rescue' asthma inhalers." Our pharmacist group particularly emphasized the patient errors characterizing medication adherence.

\section{Mental Errors}

The mental errors most proximate to action errors are memory errors, mindfulness errors, and misjudgments. Patient memory errors include forgetfulness and misrecall of information. Mindfulness errors are errors of perception associated with the amount and nature of patients' attentiveness. These errors can include inattention through failure to notice or incorrect recognition, and overattentiveness as through "hypochondriacal behavior." Misjudgments include such errors of assessment as a failure to check, monitor, or record when asked; a wrong judgment as, for example, "stopping medication just because you feel better"; and unrealistic expectations by patients who expect too much or too little of themselves or others. Patients and professionals each acknowledged these types of errors, although the patients more than the professionals referred to mindfulness errors.

Two more distal sets of mental errors are identified: knowledge deficits and patient attitudes not conducive to health. The former errors comprise knowledge errors, such as low literacy; comprehension errors; and errors of logic, such as "assuming that [I] must be OK because [I'm] feeling good." The attitudinal errors include selfishness through excessive pride, dishonesty, self-pity, and hedonism, as with "taking medicines for recreational use"; self-neglect, as through excessive selflessness and lack of self-regard; carelessness through inattention, thoughtlessness, excessive risk taking, apathy, and lack of reliability; distrust owing to disbelief, fearfulness, and uncooperativeness; anger in the face of impatience and intolerance; and a sacrificing of health needs to other priorities. The patient groups, much more often than the professional groups, perceived all these attitudes as potential mistakes.

\section{DISCUSSION}

Our study complements taxonomies of medical error by reporting the first taxonomy of errors that patients can make, alone or with others. Our taxonomy is an exploratory and descriptive framework of the chain of patient mental events that can contribute to 3 categories of errant actions by patients: namely, attendance errors, assertion errors, and adherence errors. Eleven groups of patients and health professionals contributed to the development of the taxonomy, the content of which is relevant to primary care.

Compared with the taxonomies of medical error in family practice, which reflect staff observation ${ }^{4-7}$ and incidents harming patients, ${ }^{8}$ our taxonomy emphasizes human errors (patient errors) more than health system and technical errors in the delivery of care. This taxonomy suggests how patients can contribute to the medical errors that patients have previously attributed to breakdowns in the clinician-patient relationship and in access to clinicians. ${ }^{11}$ In doing so, it identifies how variations in patient adherence may be errors. ${ }^{36,37}$ As noted above, however, whether nonadherence is an error in a given situation depends on the intentions of the patient. Our taxonomy also discerns potential errors in events that others have conceptualized as causes of suboptimal health, such as memory lapses ${ }^{20}$ and low health literacy. ${ }^{21}$ Our patient groups were more willing than our groups of health care professionals to consider as potential mistakes the patient attitudes that are not conducive to health.

\section{Strengths}

This study moves beyond a location-centric discourse on clinician and system error in medical settings to one that respects the capacity of patients-as people, consumers, and coproducers of care-to contribute to and avoid error. It also gives a voice on error to patients as well as health professionals. Calls to listen to and take account of the patient perspective have been poorly heeded by previous taxonomies of medical error. This oversight is unfortunate because patients have a valid perspective on error, ${ }^{13}$ and in this study, as in a hospital-based study, ${ }^{38}$ patients defined medical errors more broadly than have clinicians characterized clinical definitions of medical error.

\section{Limitations}

Our taxonomy elucidates only the errors to which patients can contribute. The extent to which these potential errors mirror actual errors is unknown. The categories of patient errors also overlap. For example, "leaving an illness too long [before seeing the doctor]" could reflect inattention, misjudgments, lack of knowledge, carelessness, or self-neglect.

It was beyond the scope of our framework to incorporate conditions extrinsic to patients. These conditions are not necessarily errors or events that patients 
control. Indeed, it is debatable whether mental factors in errors are errors in themselves or merely conditions potentially conducive to error. Our taxonomy reports what our participants construe as errors because our study question gave them the scope to define mistakes in their own way, which framed what they told us. The taxonomy also risks confusing error with blame. For example, forgetfulness as a result of cognitive impairment may impose no moral responsibility but may be an error because it deviates from correctness. It is paternalistic to suggest that patients necessarily are, or are not, morally responsible for their errors.

Sampling only 1 local community afforded a more narrow perspective than might have been obtained by accessing different communities. Although we included a group with high health literacy, we did not access the experiences and insights of affluent patients in an advantaged community where patient errors could be distinctively different. Nor did we include patients who might have avoided our meetings, such as very shy patients. We also did not access the perspective of clinicians in secondary and tertiary health care, although 1 participant held a senior position in integrated health care.

\section{Implications}

Our taxonomy is an early attempt to understand and recognize how patients may err and what it is that clinicians should aim to influence to help patients act safely. It is a preliminary classification that highlights and supports the need for further research to use a range of methods to elaborate and continue to discern the types of errors that patients can influence. It takes a first step in respecting that patients can help to define what error is, make mistakes, and respond to opportunities for safe patient actions, such as safe attendance, safe assertion of thoughts, and safe adherence to treatments. Other taxonomies of patient error could be developed in other health care settings.

Our study also shows how patients, clinicians, and systems can cocreate errors. For example, many potential errors, such as refusal of clinical investigations, originate in the process of patient-clinician interaction, which is itself conditioned by structures in the health system. There is a need, therefore, to move beyond seeing patient, clinician, and system errors as separate categories of error, since they are interdependent rather than mutually exclusive. An important next step may be research that attempts to describe and understand how the complex interactions of patients, clinicians, and systems can create and reduce errors. Doing so would support an inclusive and integrated analysis of, and approach to managing, patient safety events, which crosscut people, settings, and systems.
To read or post commentaries in response to this article, see it online at http://www.annfammed.org/cgi/content/full/7/3/223.

Submitted April 16, 2008; submitted, revised August 5, 2008; accepted August 15, 2008.

Key words: Safety; classification; patients; primary health care

Funding support: This research was funded by the Research Committee of the University of Auckland, Auckland, New Zealand.

Acknowledgments: We thank the University of Auckland Research Committee for funding this research, and the reviewers and editors for their helpful suggestions. We are grateful to the Glen Innes community for embracing this project in the spirit of community-campus collaboration.

\section{References}

1. Brennan TA. The Institute of Medicine report on medical errorscould it do harm? N Engl J Med. 2000;342(15):1123-1125.

2. Thomas EJ, Petersen LA. Measuring errors and adverse events in health care. J Gen Intern Med. 2003;18(1):61-67.

3. Ely JW, Levinson W, Elder NC, Mainous AG, Vinson DC. Perceived causes of family physician errors. J Fam Pract. 1995;40(4):337-344.

4. Rubin G, George A, Chinn DJ, Richardson C. Errors in general practice: development of an error classification and pilot study of a method for detecting errors. Qual Saf Health Care. 2003;12(6): 443-447.

5. Dovey SM, Meyers DS, Phillips RL Jr, et al. A preliminary taxonomy of medical errors in family practice. Qual Saf Health Care. 2002;11(3):233-238.

6. Makeham MA, Dovey SM, County M, Kidd MR. An international taxonomy for errors in general practice: a pilot study. Med J Aust. 2002;177(2):68-72.

7. Makeham MA, Stromer S, Bridges-Webb C, et al. Patient safety events reported in general practice: a taxonomy. Qual Saf Health Care. 2008;17(1):53-57.

8. Bhasale AL, Miller GC, Reid SE, Britt HC. Analysing potential harm in Australian general practice: an incident-monitoring study. Med J Aust. 1998;169(2):73-76.

9. Elder NC, Dovey SM. Classification of medical errors and preventable adverse events in primary care: a synthesis of the literature. J Fam Pract. 2002;51(11):927-932.

10. Sandars J, Esmail A. The frequency and nature of medical error in primary care: understanding the diversity across studies. Fam Pract. 2003;20(3):231-236.

11. Kuzel AJ, Woolf SH, Gilchrist VJ, et al. Patient reports of preventable problems and harms in primary health care. Ann Fam Med. 2004;2(4):333-340.

12. Agoritsas T, Bovier P, Perneger T. Patient reports of undesirable events during hospitalisation. J Gen Intern Med. 2005;20(10):922-928.

13. Vincent CA, Coulter A. Patient safety: what about the patient? Qual Saf Health Care. 2002;11(1):76-80.

14. Kohn LT, Corrigan JM, Donaldson MS. To Err is Human: Building a Safer Health System. Washington DC: National Academy Press, 2000.

15. Kornstad S, Boye NP. Patient errors and preference with regard to the use of a bronchodilator spray and feneterol (Berotec) powder. Eur J Respir Dis Suppl. 1983;130:12-16.

16. Sparr LF, Moffitt MC, Ward MF. Missed psychiatric appointments: who returns and who stays away. Am J Psychiatry. 1993;150(5): 801-805. 
17. Redelmeier DA, Schull MJ, Hux JE, Tu JV, Ferris LE. Problems for clinical judgment: 1 . Eliciting an insightful history of present illness. CMAJ. 2001;164(5):647-651.

18. Kuhn GJ. Diagnostic errors. Acad Emerg Med. 2002;9(7):740-750.

19. Lundeen JM, Souba WW, Hollenbeak CS. Sources of error in delayed payment of physician claims. Fam Med. 2003;35(5):355-359

20. Kessels RP. Patients' memory for medical information. J R Soc Med. 2003;96(5):219-222.

21. Hironaka LK, Paasche-Orlow MK. The implications of health literacy on patient-provider communication. Arch Dis Child. 2008;93(5): 428-432

22. Woods DM, Johnson J, Holl JL, et al. Anatomy of a patient safety event: a pediatric patient safety taxonomy. Qual Saf Health Care. 2005;14(6):422-427.

23. Law Reform Commission of Victoria. Informed Decisions about Medical Procedures. Doctor and Patient Studies. Melbourne: Law Reform Commission; 1989.

24. Green LA, Fryer GE Jr, Yawn BP, Lanier D, Dovey SM. The ecology of medical care revisited. N Engl J Med. 2001;344(26):2021-2025.

25. Dovey SM, Green LE, Phillips RA, Fryer EL. The patient safety grid-toxic cascades in health care settings. Am Fam Physician. 2000;63(6):1047.

26. Buetow S, Elwyn G. Patient safety and patient error. Lancet. 2007; 369(9556):158-161.

27. Reason J. Human Error. Cambridge: Cambridge University Press; 2002.

28. Zhang J, Patel VL, Johnson TR, Shortliffe EH. A cognitive taxonomy of medical errors. J Biomed Inform. 2004;37(3):193-204.
29. Donovan JL, Blake DR. Patient non-compliance: deviance or reasoned decision-making. Soc Sci Med. 1992;34(5):507-513.

30. Dovey S. Patient-centred professionalism and population health: negotiating the tension. N Z Fam Physician. 2008;35(3):154-155.

31. Chang A, Schyve PM, Croteau RJ, O'Leary DS, Loeb JM. The JCAHO patient safety event taxonomy: a standardized terminology and classification schema for near misses and adverse events. Int J Qual Health Care. 2005;17(2):95-105.

32. Woods A, Doan-Johnson S. Executive summary: toward a taxonomy of nursing practice errors. Nurs Manage. 2002;33(10):45-48.

33. Gallagher M, Hares T, Spencer J, Bradshaw C, Webb I. The nominal group technique: a research tool for general practice. Fam Pract. 1993;10(1):76-81

34. Potter M, Gordon S, Hamer P. The nominal group technique: a useful consensus methodology in physiotherapy research. NZ J Physiotherapy. 2004;32(3):126-130.

35. Thomas D. A general inductive approach for analyzing qualitative evaluation data. Am J Eval. 2006;27(2):1-10.

36. Barber N. Should we consider non-compliance a medical error? Qual Saf Health Care. 2002;11(1):81-84.

37. Lehane $\mathrm{E}, \mathrm{McCarthy} \mathrm{G}$. Intentional and unintentional medication non-adherence: a comprehensive framework for clinical research and practice? A discussion paper. Int J Nurs Stud. 2007;44(8):1468-1477.

38. Burroughs TE, Waterman AD, Gallagher TH, et al. Patients' concerns about medical errors during hospitalization. Jt Comm J Qual Patient Saf. 2007;33(1):5-14 